\title{
Eye Contact Communication System between Mobile Robots Using Invisible Code Display
}

\author{
Takeru Furukawa and Kunio Sakamoto \\ Department of Intelligence and Informatics, Konan University \\ 8-9-1 Okamoto, Higashinada, Kobe 658-8501, Japan \\ kunio@konan-u.ac.jp
}

\begin{abstract}
The authors have been developing the mobile robots which can cooperation between robots. The robots should communicate with each other in order to cooperate together. Therefore, the inter communication between robots is very important problem to be solved. These robots generally utilize wireless transmission system. The transmission sets send and receive on the same frequency or channel to establish the radio communication. This is called working simplex. The robots cannot start communication if both sets use different frequency channels. It is important to perform an initial configuration for establishing the radio signal transmission at a first contact among strange mobile robots. To solve this problem, this paper describes an information transmission system using an invisible code on displays which show an expression of robot's eyes.
\end{abstract}

Keywords: 2D code, polarized invisible code, polarized light control, LCD display.

\section{Introduction}

"The eyes are eloquent as the tongue." This proverb means that one can say more with a look than with ten thousand words. In many cases, we perceive one's true intention from his or her facial expressions and gestures. This is a nonverbal communication. The nonverbal communications are usually understood as the process of communication through sending and receiving wordless messages. The eye contact is a basic and expected form of nonverbal communication as the famous quote by: "the eyes are the window of the heart, and the heart is the house for the soul." This eye contact is a meeting of the eyes between two individuals. The authors hit upon an idea that fundamental transmission can make the best use of the eye communication. In this paper, the authors propose a communication method using an invisible code which is drawn on the eyes of the robot. We have developed the special graphics display for showing expression of robot's eyes and confirmed that our proposed technique enables the mobile robots to transmit and receive information data in order to establish a cooperation environment among robots.

A printing company has developed a new technology which makes a publication embedded with invisible codes, which is made by infrared reflective pigment, or small $2 \mathrm{D}$ codes, which is made by conventional offset printing using an infrared 
printing ink. The code symbol like a QR code consists of numerous intelligent small dots that can be read by a pen device. This pattern indicates the exact positions of the pen. When touching the pattern on the paper is automatically taken. Every snapshot contains enough data to determine the exact position of the pen device. Using this position data, the interactive computer system provides us with services such as a voice reading or a touch-panel-like operation on the paper. But this technology is realized only on the paper material. The authors would like to realize communication using an invisible code on an electrical display.

\section{2D Code Contains Information Data}

QR Code is a kind of 2D symbology developed by Denso Wave in 1994. QR Code (2D Code) contains information in both the vertical and horizontal directions, whereas a bar code contains data in one direction only. QR Code is capable of 360 degrees, high speed reading. QR Code accomplishes this task through position detection patterns located at the three corners of the symbol. This ability allows the use of 2dimensional codes in a wide range of applications. QR codes onto labels are widely used in the fields of distribution and logistics. Moreover, QR Code is available for camera phones which enable new services based on QR-code (2D-barcode) input. The QR Code can store much information. The authors utilize this QR Code in order to establish the communication among the mobile robots.

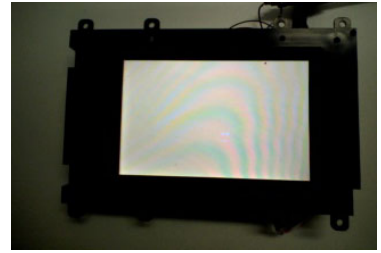

(a) view of normal camera

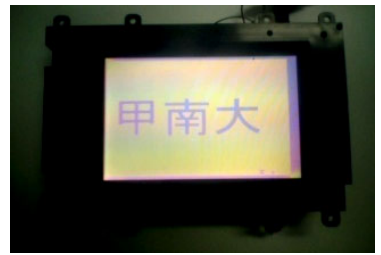

(b) thru horizontal polarizer

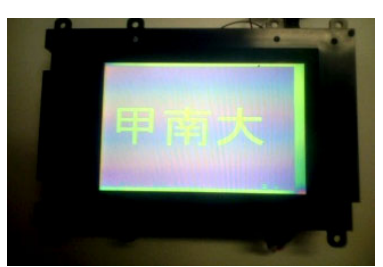

(c) thru vertical polarizer

Fig. 1. Invisible display unit

\section{Invisible Code Display}

The printing technology using a special pigment enables us to provide a publication embedded with invisible codes. This technique is useful for developing an interaction system. We want to make good use of invisible codes at an electric display as well as a paper.

To display visual information and to embed invisible additional information, the display panel needs to hide code symbols so as not to interfere with screen viewing as shown Fig. 1. So we utilize a polarized symbol image to overlap additional information on the visual screen. The polarized light wave has a useful characteristic to generate hidden images. You know you cannot perceive digits of a calculator if a polarizer is removed from an LCD, i.e., it is impossible for human's eyes to distinguish characteristics of polarization. In robot's eye expression display system 


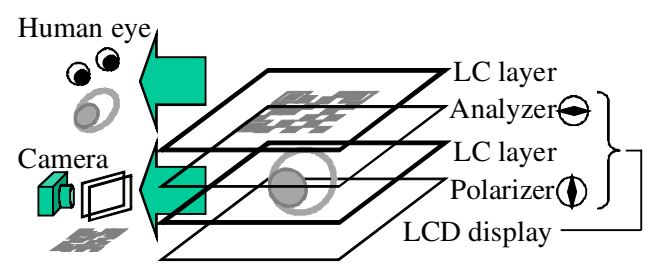

Fig. 2. Principle of generating invisible code

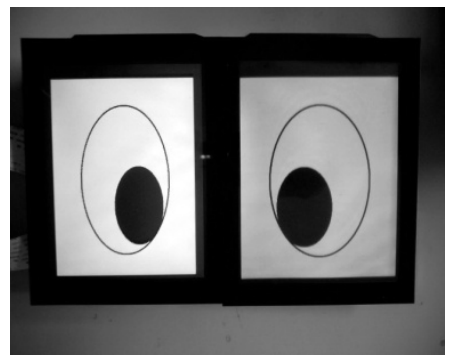

(a) expression of eye

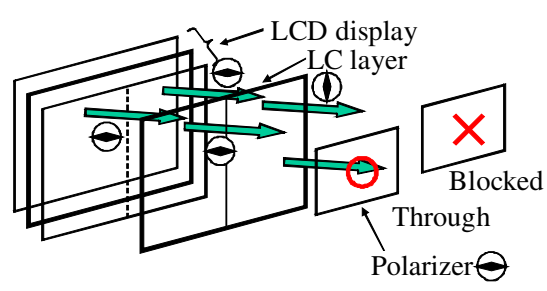

Fig. 3. Principle of hidden code detection

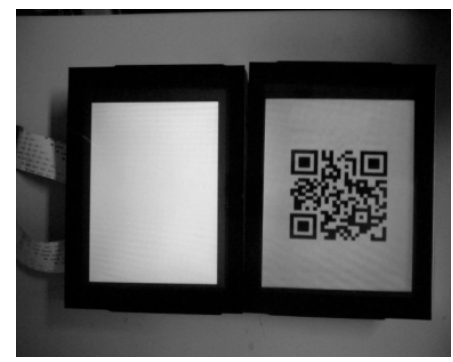

(b) additional information contained in QR code

Fig. 4. Robot eye display

using LCD panels, we utilize characteristics of polarization. As shown in Fig. 2, our proposed display system consists of a conventional LCD panel, an additional liquid crystal (LC) layer and some optical elements. LC layers can rotate the direction of the polarization axis according to the applied voltage. The LC layer sandwiched between both polarizers displays visual information. This structure functions as an LCD panel. Then this LCD panel emits the polarized light due to the existence of a surface polarizer (it is called an analyzer). Moreover, the overlaid additional LC layer changes the direction of polarization from LCD outputs. This LC layer generates invisible symbol patterns. The final LC layer outputs horizontal or vertical linear polarized light waves. This difference of orientation makes a binary symbol image. As humans cannot perceive differences of polarization, they directly watch only visual images on the viewscreen without perceiving symbol patterns. At the detection, the polarized symbol pattern images are observable through the polarizer because the polarizer blocks the wave or not according to the orientation. This enables a camera to detect the invisible code on the display panel. Humans and cameras can perceive the hidden pattern through these optical elements. So the display panels show visual images and invisible symbols simultaneously. Human's eyes can get only visual information and a code reader finds an only binary symbol pattern. Fig. 3 clearly shows the basic concept of an invisible code display. The additional LC layer turns the linear polarization from LCD outputs. We can perceive the difference of polarization using a polarizer. Assume that the polarizer rotates 90 degrees. Then the direction of polarizer converts vertical into horizontal or vice versa. This means that a detector gets an inverse image which is white and black reversed. Using this invisible code, the display system provides humans with visual information like expressions of 
robot's eyes and additional information in order to perform an initial configuration for establishing the radio signal transmission at a first contact among strange mobile robots. Fig. 4 shows the appearance of the display panel for robot's eyes. Fig. 4 (a) shows the observed image by a natural human's eye. Fig. 4 (b) shows the detected image through the polarizer.

Acknowledgments. This research is partially supported by "Grant-in-Aid for Young Scientists(B)" \#20700112 and "Scientific Research (C) (General)" \#20500481 from Ministry of Education, Culture, Sports, Science and Technology Japan(MEXT). 